\title{
Barnes' multiple Bernoulli and generalized Barnes' multiple Frobenius-Euler mixed-type polynomials
}

\section{Dae San Kim ${ }^{1}$, Taekyun Kim²*, Takao Komatsu ${ }^{3}$ and Hyuck In Kwon²}

"Correspondence: tkkim@kw.ac.kr 2Department of Mathematics, Kwangwoon University, Seoul, 139-701, Republic of Korea Full list of author information is available at the end of the article

\author{
Abstract \\ In this paper, by considering Barnes' multiple Bernoulli polynomials as well as \\ generalized Barnes' multiple Frobenius-Euler polynomials, we define and investigate \\ the mixed-type polynomials of these polynomials. From the properties of Sheffer \\ sequences of these polynomials arising from umbral calculus, we derive new and \\ interesting identities. \\ MSC: $05 \mathrm{~A} 15 ; 05 \mathrm{~A} 40 ; 11 \mathrm{~B} 68 ; 11 \mathrm{~B} 75 ; 33 \mathrm{E} 20 ; 65 \mathrm{Q} 05$
}

\section{Introduction}

In this paper, we consider the polynomials

$$
B H_{n}(x)=B H_{n}(x \mid a ; b ; \lambda ; \mu)=B H_{n}\left(x \mid a_{1}, \ldots, a_{r} ; b_{1}, \ldots, b_{s} ; \lambda_{1}, \ldots, \lambda_{s} ; \mu_{1}, \ldots, \mu_{s}\right)
$$

called Barnes' multiple Bernoulli and generalized Barnes' multiple Frobenius-Euler mixed-type polynomials, whose generating function is given by

$$
\prod_{i=1}^{r}\left(\frac{t}{e^{a_{i} t}-1}\right) \prod_{j=1}^{s}\left(\frac{1-\lambda_{j}}{e^{b_{j} t}-\lambda_{j}}\right)^{\mu_{j}} e^{x t}=\sum_{n=0}^{\infty} B H_{n}(x) \frac{t^{n}}{n !},
$$

where $a_{1}, \ldots, a_{r}, b_{1}, \ldots, b_{s}, \lambda_{1}, \ldots, \lambda_{s}, \mu_{1}, \ldots, \mu_{s} \in \mathbb{C}$ with $a_{1}, \ldots, a_{r}, b_{1}, \ldots, b_{s} \neq 0, \lambda_{1}, \ldots, \lambda_{s} \neq$ 1. When $x=0$,

$$
B H_{n}=B H_{n}(0)=B H_{n}\left(0 ; a_{1}, \ldots, a_{r} ; b_{1}, \ldots, b_{s} ; \lambda_{1}, \ldots, \lambda_{r} ; \mu_{1}, \ldots, \mu_{r}\right)
$$

are called Barnes' multiple Bernoulli and generalized Barnes' multiple Frobenius-Euler mixed-type numbers.

Recall that Barnes' multiple Bernoulli polynomials, denoted by $B_{n}\left(x \mid a_{1}, \ldots, a_{r}\right)$, are given by the generating function as

$$
\prod_{i=1}^{r}\left(\frac{t}{e^{a_{i} t}-1}\right) e^{x t}=\sum_{n=0}^{\infty} B_{n}\left(x \mid a_{1}, \ldots, a_{r}\right) \frac{t^{n}}{n !}
$$

\section{说) Springer}

02014 Kim et al:; licensee Springer. This is an Open Access article distributed under the terms of the Creative Commons Attribution License (http://creativecommons.org/licenses/by/2.0), which permits unrestricted use, distribution, and reproduction in any medium, provided the original work is properly cited. 
where $a_{1}, \ldots, a_{r} \neq 0[1,2]$. In addition, the generalized Barnes' multiple Frobenius-Euler polynomials, denoted by $H_{n}(x \mid b ; \lambda ; \mu)=H_{n}\left(x \mid b_{1}, \ldots, b_{s} ; \lambda_{1}, \ldots, \lambda_{s} ; \mu_{1}, \ldots, \mu_{s}\right)$, are given by the generating function as

$$
\prod_{j=1}^{s}\left(\frac{1-\lambda_{j}}{e^{b_{j} t}-\lambda_{j}}\right)^{\mu_{j}} e^{x t}=\sum_{n=0}^{\infty} H_{n}(x \mid b ; \lambda ; \mu) \frac{t^{n}}{n !}
$$

(see e.g. [3-7]). If $\mu_{1}=\cdots=\mu_{s}=1$, then

$$
H_{n}\left(x \mid b_{1}, \ldots, b_{s} ; \lambda_{1}, \ldots, \lambda_{s}\right)=H_{n}\left(x \mid b_{1}, \ldots, b_{s} ; \lambda_{1}, \ldots, \lambda_{s} ; 1, \ldots, 1\right)
$$

are called Barnes-type Frobenius-Euler polynomials. If further $\lambda_{1}=\cdots=\lambda_{s}=\lambda$ and $b_{1}=$ $\cdots=b_{s}=1$, then $H_{n}^{(s)}(x \mid \lambda)=H_{n}(x \mid 1, \ldots, 1 ; \lambda, \ldots, \lambda ; 1, \ldots, 1)$ are called Frobenius-Euler polynomials of order $s$ (see e.g. [8, 9]). If $\lambda_{1}=\cdots=\lambda_{s}=-1$, then $E_{n}\left(x \mid b_{1}, \ldots, b_{s} ; \mu_{1}, \ldots, \mu_{s}\right)=$ $H_{n}\left(x \mid b_{1}, \ldots, b_{s} ; 1, \ldots, 1 ; \mu_{1}, \ldots, \mu_{s}\right)$ are called generalized Barnes-type Euler polynomials. These polynomials arise naturally in connection with the study of Barnes-type Peters polynomials. Peters polynomials were mentioned in $[10, \mathrm{p} .128]$ and were investigated in e.g. [11].

In this paper, by considering Barnes' multiple Bernoulli polynomials as well as generalized Barnes' multiple Frobenius-Euler polynomials, we define and investigate the mixedtype polynomials of these polynomials. From the properties of Sheffer sequences of these polynomials arising from umbral calculus, we derive new and interesting identities.

\section{Umbral calculus}

Let $\mathbb{C}$ be a complex number field and let $\mathcal{F}$ be the set of all formal power series in the variable $t$ :

$$
\mathcal{F}=\left\{f(t)=\sum_{k=0}^{\infty} \frac{a_{k}}{k !} t^{k} \mid a_{k} \in \mathbb{C}\right\} .
$$

Let $\mathbb{P}=\mathbb{C}[x]$ and let $\mathbb{P}^{*}$ be the vector space of all linear functionals on $\mathbb{P} .\langle L \mid p(x)\rangle$ is the action of the linear functional $L$ on the polynomial $p(x)$, and we recall that the vector space operations on $\mathbb{P}^{*}$ are defined by $\langle L+M \mid p(x)\rangle=\langle L \mid p(x)\rangle+\langle M \mid p(x)\rangle,\langle c L \mid p(x)\rangle=c\langle L \mid p(x)\rangle$, where $c$ is a complex constant in $\mathbb{C}$. For $f(t) \in \mathcal{F}$, let us define the linear functional on $\mathbb{P}$ by setting

$$
\left\langle f(t) \mid x^{n}\right\rangle=a_{n} \quad(n \geq 0) .
$$

In particular,

$$
\left\langle t^{k} \mid x^{n}\right\rangle=n ! \delta_{n, k} \quad(n, k \geq 0)
$$

where $\delta_{n, k}$ is the Kronecker symbol.

For $f_{L}(t)=\sum_{k=0}^{\infty} \frac{\left\langle L \mid x^{k}\right\rangle}{k !} t^{k}$, we have $\left\langle f_{L}(t) \mid x^{n}\right\rangle=\left\langle L \mid x^{n}\right\rangle$. That is, $L=f_{L}(t)$. The map $L \mapsto f_{L}(t)$ is a vector space isomorphism from $\mathbb{P}^{*}$ onto $\mathcal{F}$. Henceforth, $\mathcal{F}$ denotes both the algebra of formal power series in $t$ and the vector space of all linear functionals on $\mathbb{P}$, and so an 
element $f(t)$ of $\mathcal{F}$ will be thought of as both a formal power series and a linear functional. We call $\mathcal{F}$ umbral algebra, and umbral calculus is the study of umbral algebra. The order $O(f(t))$ of a power series $f(t)(\neq 0)$ is the smallest integer $k$ for which the coefficient of $t^{k}$ does not vanish. If $O(f(t))=1$, then $f(t)$ is called a delta series; if $O(f(t))=0$, then $f(t)$ is called an invertible series. For $f(t), g(t) \in \mathcal{F}$ with $O(f(t))=1$ and $O(g(t))=0$, there exists a unique sequence $s_{n}(x)\left(\operatorname{deg} s_{n}(x)=n\right)$ such that $\left\langle g(t) f(t)^{k} \mid s_{n}(x)\right\rangle=n ! \delta_{n, k}$ for $n, k \geq 0$ [10, Theorem 2.3.1]. Such a sequence $s_{n}(x)$ is called the Sheffer sequence for $(g(t), f(t))$ which is denoted by $s_{n}(x) \sim(g(t), f(t))$.

For $f(t), g(t) \in \mathcal{F}$ and $p(x) \in \mathbb{P}$, we have

$$
\langle f(t) g(t) \mid p(x)\rangle=\langle f(t) \mid g(t) p(x)\rangle=\langle g(t) \mid f(t) p(x)\rangle
$$

and

$$
f(t)=\sum_{k=0}^{\infty}\left\langle f(t) \mid x^{k}\right\rangle \frac{t^{k}}{k !}, \quad p(x)=\sum_{k=0}^{\infty}\left\langle t^{k} \mid p(x)\right\rangle \frac{x^{k}}{k !},
$$

[10, Theorem 2.2.5]. Thus, by (8), we get

$$
t^{k} p(x)=p(x)=\frac{d^{k} p(x)}{d x^{k}} \quad \text { and } \quad e^{y t} p(x)=p(x+y) .
$$

Sheffer sequences are characterized in the generating function [10, Theorem 2.3.4].

Lemma 1 The sequence $s_{n}(x)$ is Sheffer for $(g(t), f(t))$ if and only if

$$
\frac{1}{g(\bar{f}(t))} e^{y \bar{f}(t)}=\sum_{k=0}^{\infty} \frac{s_{k}(y)}{k !} t^{k} \quad(y \in \mathbb{C})
$$

where $\bar{f}(t)$ is the compositional inverse of $f(t)$.

For $s_{n}(x) \sim(g(t), f(t))$, we have the following equations [10, Theorem 2.3.7, Theorem 2.3.5, Theorem 2.3.9]:

$$
\begin{aligned}
& f(t) s_{n}(x)=n s_{n-1}(x) \quad(n \geq 0), \\
& s_{n}(x)=\sum_{j=0}^{n} \frac{1}{j !}\left\langle g(\bar{f}(t))^{-1} \bar{f}(t)^{j}\left|x^{n}\right| x^{j},\right. \\
& s_{n}(x+y)=\sum_{j=0}^{n}\left(\begin{array}{c}
n \\
j
\end{array}\right) s_{j}(x) p_{n-j}(y),
\end{aligned}
$$

where $p_{n}(x)=g(t) s_{n}(x)$.

Assume that $p_{n}(x) \sim(1, f(t))$ and $q_{n}(x) \sim(1, g(t))$. Then the transfer formula [10, Corollary 3.8.2] is given by

$$
q_{n}(x)=x\left(\frac{f(t)}{g(t)}\right)^{n} x^{-1} p_{n}(x) \quad(n \geq 1) .
$$


For $s_{n}(x) \sim(g(t), f(t))$ and $r_{n}(x) \sim(h(t), l(t))$, assume that

$$
s_{n}(x)=\sum_{m=0}^{n} C_{n, m} r_{m}(x) \quad(n \geq 0)
$$

Then we have [10, p.132]

$$
C_{n, m}=\frac{1}{m !}\left\langle\frac{h(\bar{f}(t))}{g(\bar{f}(t))} l(\bar{f}(t))^{m} \mid x^{n}\right\rangle .
$$

\section{Main results}

From definitions (2), (3) and (1), $B_{n}\left(x \mid a_{1}, \ldots, a_{r}\right), H_{n}\left(x \mid b_{1}, \ldots, b_{s} ; \lambda_{1}, \ldots, \lambda_{s} ; \mu_{1}, \ldots, \mu_{s}\right)$ and $B H_{n}\left(x \mid a_{1}, \ldots, a_{r} ; b_{1}, \ldots, b_{s} ; \lambda_{1}, \ldots, \lambda_{s} ; \mu_{1}, \ldots, \mu_{s}\right)$ are the Appell sequences for

$$
\prod_{i=1}^{r}\left(\frac{e^{a_{i} t}-1}{t}\right), \quad \prod_{j=1}^{s}\left(\frac{e^{b_{j} t}-\lambda_{j}}{1-\lambda_{j}}\right)^{\mu_{j}} \text { and } \prod_{i=1}^{r}\left(\frac{e^{a_{i} t}-1}{t}\right) \prod_{j=1}^{s}\left(\frac{e^{b_{j} t}-\lambda_{j}}{1-\lambda_{j}}\right)^{\mu_{j}},
$$

respectively. So,

$$
\begin{aligned}
& B_{n}\left(x \mid a_{1}, \ldots, a_{r}\right) \sim\left(\sum_{i=1}^{r}\left(\frac{e^{a_{i} t}-1}{t}\right), t\right), \\
& H_{n}\left(x \mid b_{1}, \ldots, b_{s} ; \lambda_{1}, \ldots, \lambda_{s} ; \mu_{1}, \ldots, \mu_{s}\right) \sim\left(\prod_{j=1}^{s}\left(\frac{e^{b_{j} t}-\lambda_{j}}{1-\lambda_{j}}\right)^{\mu_{j}}, t\right), \\
& B H_{n}\left(x \mid a_{1}, \ldots, a_{r} ; b_{1}, \ldots, b_{s} ; \lambda_{1}, \ldots, \lambda_{s} ; \mu_{1}, \ldots, \mu_{s}\right) \\
& \quad \sim\left(\prod_{i=1}^{r}\left(\frac{e^{a_{i} t}-1}{t}\right) \prod_{j=1}^{s}\left(\frac{e^{b_{j} t}-\lambda_{j}}{1-\lambda_{j}}\right)^{\mu_{j}}, t\right) .
\end{aligned}
$$

In particular,

$$
\begin{aligned}
& t B_{n}(x \mid a)=\frac{d}{d x} B_{n}(x \mid a)=n B_{n-1}(x \mid a), \\
& t H_{n}(x \mid b ; \lambda ; \mu)=\frac{d}{d x} H_{n}(x \mid b ; \lambda ; \mu)=n H_{n-1}(x \mid b ; \lambda ; \mu), \\
& t B H_{n}(x \mid a ; b ; \lambda ; \mu)=\frac{d}{d x} B H_{n}(x \mid a ; b ; \lambda ; \mu)=n B H_{n-1}(x \mid a ; b ; \lambda ; \mu),
\end{aligned}
$$

where $a=\left(a_{1}, \ldots, a_{r}\right), b=\left(b_{1}, \ldots, b_{s}\right), \lambda=\left(\lambda_{1}, \ldots, \lambda_{s}\right)$ and $\mu=\left(\mu_{1}, \ldots, \mu_{s}\right)$.

\subsection{Explicit expressions}

Let $(n)_{j}=n(n-1) \cdots(n-j+1)(j \geq 1)$ with $(n)_{0}=1$. The (signed) Stirling numbers of the first kind $S_{1}(n, m)$ are defined by

$$
(x)_{n}=\sum_{m=0}^{n} S_{1}(n, m) x^{m}
$$


Notice that

$$
(x)_{n} \sim\left(1, e^{t}-1\right) .
$$

Theorem 1 We have

$$
\begin{aligned}
B H_{n}(x \mid a ; b ; \lambda ; \mu) & =\sum_{j=0}^{n}\left(\begin{array}{l}
n \\
j
\end{array}\right) B H_{n-j} x^{j} \\
& =\sum_{l=0}^{n}\left(\begin{array}{l}
n \\
l
\end{array}\right) B_{n-l}(a) H_{l}(x \mid b ; \lambda ; \mu) \\
& =\sum_{l=0}^{n}\left(\begin{array}{l}
n \\
l
\end{array}\right) H_{n-l}(b ; \lambda ; \mu) B_{l}(x \mid a) .
\end{aligned}
$$

Proof By (11) with (16), we get

$$
\begin{aligned}
& \left\langle g(\bar{f}(t))^{-1} \bar{f}(t)^{j} \mid x^{n}\right\rangle \\
& =\left\langle\prod_{i=1}^{r}\left(\frac{t}{e^{a_{i} t}-1}\right) \prod_{j=1}^{s}\left(\frac{1-\lambda_{j}}{e^{b_{j} t}-\lambda_{j}}\right)^{\mu_{j}} t^{j} \mid x^{n}\right\rangle \\
& =\left\langle\prod_{i=1}^{r}\left(\frac{t}{e^{a_{i} t}-1}\right) \prod_{j=1}^{s}\left(\frac{1-\lambda_{j}}{e^{b_{j} t}-\lambda_{j}}\right)^{\mu_{j}} \mid t^{j} x^{n}\right\rangle \\
& =(n)_{j}\left\langle\prod_{i=1}^{r}\left(\frac{t}{e^{a_{i} t}-1}\right) \prod_{j=1}^{s}\left(\frac{1-\lambda_{j}}{e^{b_{j} t}-\lambda_{j}}\right)^{\mu_{j}} \mid x^{n-j}\right\rangle \\
& \quad=(n)_{j}\left\langle\sum_{i=0}^{\infty} B H_{i} \frac{t^{i}}{i !} \mid x^{n-j}\right\rangle \\
& \quad=(n)_{j} B H_{n-j} .
\end{aligned}
$$

Thus, we obtain identity (18).

Next,

$$
\begin{aligned}
B H_{n}(y \mid a ; b ; \lambda ; \mu) & =\left\langle\sum_{i=0}^{\infty} B H_{i}(y \mid a ; b ; \lambda ; \mu) \frac{t^{i}}{i !} \mid x^{n}\right\rangle \\
& =\left\langle\prod_{i=1}^{r}\left(\frac{t}{e^{a_{i} t}-1}\right) \prod_{j=1}^{s}\left(\frac{1-\lambda_{j}}{e^{b_{j} t}-\lambda_{j}}\right)^{\mu_{j}} e^{y t} \mid x^{n}\right\rangle \\
& =\left\langle\prod_{i=1}^{r}\left(\frac{t}{e^{a_{i} t}-1}\right) \mid \prod_{j=1}^{s}\left(\frac{1-\lambda_{j}}{e^{b_{j} t}-\lambda_{j}}\right)^{\mu_{j}} e^{y t} x^{n}\right\rangle \\
& =\left\langle\prod_{i=1}^{r}\left(\frac{t}{e^{a_{i} t}-1}\right) \mid \sum_{l=0}^{\infty} H_{l}(y \mid b ; \lambda ; \mu) \frac{t^{l}}{l !} x^{n}\right\rangle \\
& =\sum_{l=0}^{n}\left(\begin{array}{l}
n \\
l
\end{array}\right) H_{l}(y \mid b ; \lambda ; \mu)\left\langle\prod_{i=1}^{r}\left(\frac{t}{e^{a_{i} t}-1}\right) \mid x^{n-l}\right\rangle
\end{aligned}
$$




$$
\begin{aligned}
& =\sum_{l=0}^{n}\left(\begin{array}{l}
n \\
l
\end{array}\right) H_{l}(y \mid b ; \lambda ; \mu)\left\langle\sum_{i=0}^{\infty} B_{i}(a) \frac{t^{i}}{i !} \mid x^{n-l}\right\rangle \\
& =\sum_{l=0}^{n}\left(\begin{array}{l}
n \\
l
\end{array}\right) H_{l}(y \mid b ; \lambda ; \mu) B_{n-l}(a) .
\end{aligned}
$$

Thus, we obtain (19).

Finally, we obtain that

$$
\begin{aligned}
B H_{n}(y \mid a ; b ; \lambda ; \mu) & =\left\langle\sum_{i=0}^{\infty} B H_{i}(y \mid a ; b ; \lambda ; \mu) \frac{t^{i}}{i !} \mid x^{n}\right\rangle \\
& =\left\langle\prod_{i=1}^{r}\left(\frac{t}{e^{a_{i} t}-1}\right) \prod_{j=1}^{s}\left(\frac{1-\lambda_{j}}{e^{b_{j} t}-\lambda_{j}}\right)^{\mu_{j}} e^{y t} \mid x^{n}\right\rangle \\
& =\left\langle\prod_{j=1}^{s}\left(\frac{1-\lambda_{j}}{e^{b_{j} t}-\lambda_{j}}\right)^{\mu_{j}} \mid \prod_{i=1}^{r}\left(\frac{t}{e^{a_{i} t}-1}\right) e^{y t} x^{n}\right\rangle \\
& =\left\langle\prod_{j=1}^{s}\left(\frac{1-\lambda_{j}}{e^{b_{j} t}-\lambda_{j}}\right)^{\mu_{j}} \mid \sum_{l=0}^{\infty} B_{l}(y \mid a) \frac{t^{l}}{l !} x^{n}\right\rangle \\
& =\sum_{l=0}^{n}\left(\begin{array}{l}
n \\
l
\end{array}\right) B_{l}(y \mid a)\left\langle\prod_{j=1}^{s}\left(\frac{1-\lambda_{j}}{e^{b_{j} t}-\lambda_{j}}\right)^{\mu_{j}} \mid x^{n-l}\right\rangle \\
& =\sum_{l=0}^{n}\left(\begin{array}{l}
n \\
l
\end{array}\right) B_{l}(y \mid a)\left\langle\sum_{i=0}^{\infty} H_{i}(b ; \lambda ; \mu) \frac{t^{i}}{i !} \mid x^{n-l}\right\rangle \\
& =\sum_{l=0}^{n}\left(\begin{array}{l}
n \\
l
\end{array}\right) B_{l}(y \mid a) H_{n-l}(b ; \lambda ; \mu) .
\end{aligned}
$$

Thus, we get identity (20).

\subsection{The Sheffer identity}

\section{Theorem 2}

$$
B H_{n}(x+y \mid a ; b ; \lambda ; \mu)=\sum_{j=0}^{n}\left(\begin{array}{l}
n \\
j
\end{array}\right) B H_{j}(x \mid a ; b ; \lambda ; \mu) y^{n-j} .
$$

Proof By (16) with

$$
\begin{aligned}
p_{n}(x) & =\prod_{i=1}^{r}\left(\frac{e^{a_{i} t}-1}{t}\right) \prod_{j=1}^{s}\left(\frac{e^{b_{j} t}-\lambda_{j}}{1-\lambda_{j}}\right)^{\mu_{j}} B H_{n}(x \mid a ; b ; \lambda ; \mu) \\
& =x^{n} \sim(1, t),
\end{aligned}
$$

using (12), we have (21). 


\subsection{Recurrence}

\section{Theorem 3}

$$
\begin{aligned}
(1 & \left.-\frac{r}{n+1}\right) B H_{n+1}(x \mid a ; b ; \lambda ; \mu) \\
= & x B H_{n}(x \mid a ; b ; \lambda ; \mu)-\frac{1}{n+1} \sum_{i=1}^{r} a_{i} B H_{n+1}\left(x+a_{i} \mid a, a_{i} ; b ; \lambda ; \mu\right) \\
& \quad-\sum_{j=1}^{s} \frac{\mu_{j} b_{j}}{1-\lambda_{j}} B H_{n}\left(x+b_{j} \mid a ; b ; \lambda ; \mu+e_{j}\right) .
\end{aligned}
$$

Remark When $n=r-1$, as the left-hand side of (22) is equal to 0 , we have

$$
\begin{aligned}
x B H_{r-1}(x \mid a ; b ; \lambda ; \mu)= & \frac{1}{r} \sum_{i=1}^{r} a_{i} B H_{r}\left(x+a_{i} \mid a, a_{i} ; b ; \lambda ; \mu\right) \\
& +\sum_{j=1}^{s} \frac{\mu_{j} b_{j}}{1-\lambda_{j}} B H_{r-1}\left(x+b_{j} \mid a ; b ; \lambda ; \mu+e_{j}\right) .
\end{aligned}
$$

Proof By applying

$$
s_{n+1}(x)=\left(x-\frac{g^{\prime}(t)}{g(t)}\right) \frac{1}{f^{\prime}(t)} s_{n}(x)
$$

[10, Corollary 3.7.2] with (16), we get

$$
B H_{n+1}(x \mid a ; b ; \lambda ; \mu)=x B H_{n}(x \mid a ; b ; \lambda ; \mu)-\frac{g^{\prime}(t)}{g(t)} B H_{n}(x \mid a ; b ; \lambda ; \mu) .
$$

Since

$$
\begin{aligned}
\frac{g^{\prime}(t)}{g(t)} & =(\ln g(t))^{\prime}=\left(\sum_{i=1}^{r} \ln \left(e^{a_{i} t}-1\right)-r \ln t+\sum_{j=1}^{s} \mu_{j} \ln \left(e^{b_{j} t}-\lambda_{j}\right)-\sum_{j=1}^{s} \mu_{j} \ln \left(1-\lambda_{j}\right)\right)^{\prime} \\
& =\sum_{i=1}^{r} \frac{a_{i} e^{a_{i} t}}{e^{a_{i} t}-1}-\frac{r}{t}+\sum_{j=1}^{s} \frac{\mu_{j} b_{j} e^{b_{j} t}}{e^{b_{j} t}-\lambda_{j}}=\frac{1}{t} \sum_{i=1}^{r}\left(\frac{a_{i} t e^{a_{i} t}}{e^{a_{i} t}-1}-1\right)+\sum_{j=1}^{s} \frac{\mu_{j} b_{j} e^{b_{j} t}}{e^{b_{j} t}-\lambda_{j}}
\end{aligned}
$$

and

$$
\sum_{i=1}^{r}\left(\frac{a_{i} t e^{a_{i} t}}{e^{a_{i} t}-1}-1\right)=\frac{1}{2}\left(\sum_{i=1}^{r} a_{i}\right) t+\cdots
$$

is a series with order at least one, we have

$$
\begin{aligned}
& \frac{g^{\prime}(t)}{g(t)} B H_{n}(x \mid a ; b ; \lambda ; \mu) \\
& \quad=\left(\frac{1}{t} \sum_{i=1}^{r}\left(\frac{a_{i} t e^{a_{i} t}}{e^{a_{i} t}-1}-1\right)+\sum_{j=1}^{s} \frac{\mu_{j} b_{j} e^{b_{j} t}}{1-\lambda_{j}} \frac{1-\lambda_{j}}{e^{b_{j} t}-\lambda_{j}}\right) B H_{n}(x \mid a ; b ; \lambda ; \mu)
\end{aligned}
$$




$$
\begin{aligned}
= & \frac{1}{t} \sum_{i=1}^{r}\left(\frac{a_{i} t e^{a_{i} t}}{e^{a_{i} t}-1}-1\right) B H_{n}(x \mid a ; b ; \lambda ; \mu) \\
& +\sum_{j=1}^{s} \frac{\mu_{j} b_{j} e^{a_{j} t}}{1-\lambda_{j}} \prod_{i=1}^{r}\left(\frac{t}{e^{a_{i} t}-1}\right) \frac{1-\lambda_{j}}{e^{b_{j} t}-\lambda_{j}} \prod_{j=1}^{s}\left(\frac{1-\lambda_{j}}{e^{b_{j} t}-\lambda_{j}}\right)^{\mu_{j}} x^{n} \\
= & \frac{1}{n+1}\left(\sum_{i=1}^{r} \frac{a_{i} t e^{a_{i} t}}{e^{a_{i} t}-1}-r\right) B H_{n+1}(x \mid a ; b ; \lambda ; \mu)+\sum_{j=1}^{s} \frac{\mu_{j} b_{j}}{1-\lambda_{j}} B H_{n}\left(x+b_{j} \mid a ; b ; \lambda ; \mu+e_{j}\right) \\
= & \frac{1}{n+1} \sum_{i=1}^{r} a_{i} e^{a_{i} t} \frac{t}{e^{a_{i} t}-1} \prod_{v=1}^{r}\left(\frac{t}{e^{a_{v} t}-1}\right) \prod_{j=1}^{s}\left(\frac{1-\lambda_{j}}{e^{b_{j} t}-\lambda_{j}}\right)^{\mu_{j}} x^{n+1} \\
& -\frac{r}{n+1} B H_{n+1}(x \mid a ; b ; \lambda ; \mu)+\sum_{j=1}^{s} \frac{\mu_{j} b_{j}}{1-\lambda_{j}} B H_{n}\left(x+b_{j} \mid a ; b ; \lambda ; \mu+e_{j}\right) \\
= & \frac{1}{n+1} \sum_{i=1}^{r} a_{i} B H_{n+1}\left(x+a_{i} \mid a, a_{i} ; b ; \lambda ; \mu\right)-\frac{r}{n+1} B H_{n+1}(x \mid a ; b ; \lambda ; \mu) \\
& +\sum_{j=1}^{s} \frac{\mu_{j} b_{j}}{1-\lambda_{j}} B H_{n}\left(x+b_{j} \mid a ; b ; \lambda ; \mu+e_{j}\right) .
\end{aligned}
$$

Here $\left(a, a_{i}\right)=\left(a_{1}, \ldots, a_{r}, a_{i}\right)$ and $e_{i}=(\underbrace{0, \ldots, 0}_{i-1}, 1, \underbrace{0, \ldots, 0}_{r-i})(i=1,2, \ldots, r)$. Therefore, we obtain

$$
\begin{aligned}
(1 & \left.-\frac{r}{n+1}\right) B H_{n+1}(x \mid a ; b ; \lambda ; \mu) \\
= & x B H_{n}(x \mid a ; b ; \lambda ; \mu)-\frac{1}{n+1} \sum_{i=1}^{r} a_{i} B H_{n+1}\left(x+a_{i} \mid a, a_{i} ; b ; \lambda ; \mu\right) \\
& \quad-\sum_{j=1}^{s} \frac{\mu_{j} b_{j}}{1-\lambda_{j}} B H_{n}\left(x+b_{j} \mid a ; b ; \lambda ; \mu+e_{j}\right)
\end{aligned}
$$

which is (22).

\subsection{More relations}

Theorem 4 For $n \geq 1$, we have

$$
\begin{aligned}
B H_{n}(x \mid a ; b ; \lambda ; \mu)= & x B H_{n-1}(x \mid a ; b ; \lambda ; \mu)-\sum_{j=1}^{s} \frac{\mu_{j} b_{j}}{1-\lambda_{j}} B H_{n-1}\left(x+b_{j} \mid a ; b ; \lambda ; \mu+e_{j}\right) \\
& +\sum_{m=1}^{n} \frac{(-1)^{m-1}\left(\begin{array}{c}
n-1 \\
m-1
\end{array}\right) B_{m}}{m} B H_{n-m}(x \mid a ; b ; \lambda ; \mu) \sum_{i=1}^{r} a_{i}^{m} .
\end{aligned}
$$

Proof For $n \geq 1$, we have

$$
\begin{aligned}
B H_{n}(y \mid a ; b ; \lambda ; \mu) & =\left\langle\sum_{l=0}^{\infty} B H_{l}(y \mid a ; b ; \lambda ; \mu) \frac{t^{l}}{l !} \mid x^{n}\right\rangle \\
& =\left\langle\prod_{i=1}^{r}\left(\frac{t}{e^{a_{i} t}-1}\right) \prod_{j=1}^{s}\left(\frac{1-\lambda_{j}}{e^{b_{j} t}-\lambda_{j}}\right)^{\mu_{j}} e^{y t} \mid x^{n}\right\rangle
\end{aligned}
$$




$$
\begin{aligned}
= & \left\langle\partial_{t}\left(\prod_{i=1}^{r}\left(\frac{t}{e^{a_{i} t}-1}\right) \prod_{j=1}^{s}\left(\frac{1-\lambda_{j}}{e^{b_{j} t}-\lambda_{j}}\right)^{\mu_{j}} e^{y t}\right) \mid x^{n-1}\right\rangle \\
= & \left\langle\left(\partial_{t} \prod_{i=1}^{r}\left(\frac{t}{e^{a_{i} t}-1}\right)\right) \prod_{j=1}^{s}\left(\frac{1-\lambda_{j}}{e^{b_{j} t}-\lambda_{j}}\right)^{\mu_{j}} e^{y t} \mid x^{n-1}\right\rangle \\
& +\left\langle\prod_{i=1}^{r}\left(\frac{t}{e^{a_{i} t}-1}\right)\left(\partial_{t} \prod_{j=1}^{s}\left(\frac{1-\lambda_{j}}{e^{b_{j} t}-\lambda_{j}}\right)^{\mu_{j}}\right) e^{y t} \mid x^{n-1}\right\rangle \\
& +\left\langle\prod_{i=1}^{r}\left(\frac{t}{e^{a_{i} t}-1}\right) \prod_{j=1}^{s}\left(\frac{1-\lambda_{j}}{e^{b_{j} t}-\lambda_{j}}\right)^{\mu_{j}}\left(\partial_{t} e^{y t}\right) \mid x^{n-1}\right\rangle .
\end{aligned}
$$

The third term is

$$
y\left\langle\prod_{i=1}^{r}\left(\frac{t}{e^{a_{i} t}-1}\right) \prod_{j=1}^{s}\left(\frac{1-\lambda_{j}}{e^{b_{j} t}-\lambda_{j}}\right)^{\mu_{j}} e^{y t} \mid x^{n-1}\right\rangle=y B H_{n-1}(y \mid a ; b ; \lambda ; \mu) .
$$

Since

$$
\begin{aligned}
\partial_{t} \prod_{i=1}^{r}\left(\frac{t}{e^{a_{i} t}-1}\right) & =\sum_{i=1}^{r}\left(\frac{t}{e^{a_{i} t}-1}\right)^{\prime} \prod_{\nu \neq i} \frac{t}{e^{a_{v} t}-1} \\
& =\frac{t^{r-1}}{\prod_{\nu=1}^{r}\left(e^{a_{\nu} t}-1\right)}\left(r-\sum_{i=1}^{r} \frac{a_{i} t e^{a_{i} t}}{e^{a_{i} t}-1}\right) \\
& =\frac{t^{r-1}}{\prod_{\nu=1}^{r}\left(e^{a_{v} t}-1\right)}\left(r-\sum_{i=1}^{r} \frac{-a_{i} t}{e^{-a_{i} t}-1}\right) \\
& =\frac{t^{r-1}}{\prod_{\nu=1}^{r}\left(e^{a_{\nu} t}-1\right)}\left(r-\sum_{i=1}^{r} \sum_{m=0}^{\infty} \frac{\left(-a_{i}\right)^{m} B_{m} t^{m}}{m !}\right) \\
& =\frac{t^{r-1}}{\prod_{\nu=1}^{r}\left(e^{a_{\nu} t}-1\right)}\left(r-\sum_{m=0}^{\infty} \sum_{i=1}^{r}\left(-a_{i}\right)^{m} \frac{B_{m} t^{m}}{m !}\right) \\
& =\frac{t^{r}}{\prod_{\nu=1}^{r}\left(e^{a_{\nu} t}-1\right)} \sum_{m=1}^{\infty} \sum_{i=1}^{r} a_{i}^{m} \frac{(-1)^{m-1} B_{m}}{m !} t^{m-1},
\end{aligned}
$$

the first term is

$$
\begin{aligned}
& \left\langle\prod_{i=1}^{r}\left(\frac{t}{e^{a_{i} t}-1}\right) \prod_{j=1}^{s}\left(\frac{1-\lambda_{j}}{e^{b_{j} t}-\lambda_{j}}\right)^{\mu_{j}} e^{y t} \mid \sum_{m=1}^{\infty} \sum_{i=1}^{r} a_{i}^{m} \frac{(-1)^{m-1} B_{m}}{m !} t^{m-1} x^{n-1}\right\rangle \\
& \quad=\sum_{m=1}^{n} \frac{(-1)^{m-1}\left(\begin{array}{c}
n-1 \\
m-1
\end{array}\right) B_{m}}{m} \sum_{i=1}^{r} a_{i}^{m}\left\langle\prod_{i=1}^{r}\left(\frac{t}{e^{a_{i} t}-1}\right) \prod_{j=1}^{s}\left(\frac{1-\lambda_{j}}{e^{b_{j} t}-\lambda_{j}}\right)^{\mu_{j}} e^{y t} \mid x^{n-m}\right\rangle \\
& \quad=\sum_{m=1}^{n} \frac{(-1)^{m-1}\left(\begin{array}{c}
n-1 \\
m-1
\end{array}\right) B_{m}}{m} B H_{n-m}(y \mid a ; b ; \lambda ; \mu) \sum_{i=1}^{r} a_{i}^{m} .
\end{aligned}
$$


Since

$$
\begin{aligned}
\partial_{t} \prod_{j=1}^{s}\left(\frac{1-\lambda_{j}}{e^{b_{j} t}-\lambda_{j}}\right)^{\mu_{j}} & =\sum_{j=1}^{s}\left(\left(\frac{1-\lambda_{j}}{e^{b_{j} t}-\lambda_{j}}\right)^{\mu_{j}}\right)^{\prime} \prod_{\nu \neq j}\left(\frac{1-\lambda_{v}}{e^{b_{\nu} t}-\lambda_{\nu}}\right)^{\mu_{v}} \\
& =-\prod_{j=1}^{s}\left(\frac{1-\lambda_{j}}{e^{b_{j} t}-\lambda_{j}}\right)^{\mu_{j}} \sum_{\nu=1}^{s} \frac{\mu_{\nu} b_{v} e^{b_{v} t}}{1-\lambda_{v}} \frac{1-\lambda_{v}}{e^{b_{\nu} t}-\lambda_{v}},
\end{aligned}
$$

the second term is

$$
\begin{aligned}
& -\sum_{\nu=1}^{s} \frac{\mu_{\nu} b_{v}}{1-\lambda_{\nu}}\left\langle\prod_{i=1}^{r}\left(\frac{t}{e^{a_{i} t}-1}\right) \frac{1-\lambda_{\nu}}{e^{b_{\nu} t}-\lambda_{\nu}} \prod_{j=1}^{s}\left(\frac{1-\lambda_{j}}{e^{b_{j} t}-\lambda_{j}}\right)^{\mu_{j}} e^{\left(y+b_{v}\right) t} \mid x^{n-1}\right\rangle \\
& =-\sum_{\nu=1}^{s} \frac{\mu_{\nu} b_{v}}{1-\lambda_{\nu}} B H_{n-1}\left(y+b_{v} \mid a ; b ; \lambda ; \mu+e_{\nu}\right) .
\end{aligned}
$$

Therefore, we obtain

$$
\begin{aligned}
B H_{n}(x \mid a ; b ; \lambda ; \mu)= & x B H_{n-1}(x \mid a ; b ; \lambda ; \mu)-\sum_{j=1}^{s} \frac{\mu_{j} b_{j}}{1-\lambda_{j}} B H_{n-1}\left(x+b_{j} \mid a ; b ; \lambda ; \mu+e_{j}\right) \\
& +\sum_{m=1}^{n} \frac{(-1)^{m-1}\left(\begin{array}{c}
n-1 \\
m-1
\end{array}\right) B_{m}}{m} B H_{n-m}(x \mid a ; b ; \lambda ; \mu) \sum_{i=1}^{r} a_{i}^{m},
\end{aligned}
$$

which is identity (24).

\subsection{A relation including Bernoulli numbers}

Theorem 5 For $n-1 \geq m \geq 1$, we have

$$
\begin{aligned}
(n- & m) B H_{n-m}(a ; b ; \lambda ; \mu) \\
= & -(n-m) \sum_{j=1}^{s} \frac{\mu_{j} b_{j}}{1-\lambda_{j}} B H_{n-m-1}\left(b_{i} \mid a ; b ; \lambda ; \mu+e_{j}\right) \\
& +\sum_{l=1}^{n-m}(-1)^{l-1}\left(\begin{array}{c}
n-m \\
l
\end{array}\right) B_{l} B H_{n-m-l}(a ; b ; \lambda ; \mu) \sum_{i=1}^{r} a_{i}^{l} .
\end{aligned}
$$

Proof We shall compute

$$
\left\langle\prod_{i=1}^{r}\left(\frac{t}{e^{a_{i} t}-1}\right) \prod_{j=1}^{s}\left(\frac{1-\lambda_{j}}{e^{b_{j} t}-\lambda_{j}}\right)^{\mu_{j}} t^{m} \mid x^{n}\right\rangle
$$

in two different ways. On the one hand, it is equal to

$$
\begin{aligned}
& \left\langle\prod_{i=1}^{r}\left(\frac{t}{e^{a_{i} t}-1}\right) \prod_{j=1}^{s}\left(\frac{1-\lambda_{j}}{e^{b_{j} t}-\lambda_{j}}\right)^{\mu_{j}} \mid t^{m} x^{n}\right\rangle \\
& \quad=(n)_{m}\left\langle\prod_{i=1}^{r}\left(\frac{t}{e^{a_{i} t}-1}\right) \prod_{j=1}^{s}\left(\frac{1-\lambda_{j}}{e^{b_{j} t}-\lambda_{j}}\right)^{\mu_{j}} \mid x^{n-m}\right\rangle \\
& \quad=(n)_{m} B H_{n-m}(a ; b ; \lambda ; \mu) .
\end{aligned}
$$


On the other hand, it is equal to

$$
\begin{aligned}
\left\langle\partial_{t}\right. & \left.\prod_{i=1}^{r}\left(\frac{t}{e^{a_{i} t}-1}\right) \prod_{j=1}^{s}\left(\frac{1-\lambda_{j}}{e^{b_{j} t}-\lambda_{j}}\right)^{\mu_{j}} t^{m}\right)\left|x^{n-1}\right\rangle \\
= & \left\langle\left(\partial_{t} \prod_{i=1}^{r}\left(\frac{t}{e^{a_{i} t}-1}\right)\right) \prod_{j=1}^{s}\left(\frac{1-\lambda_{j}}{e^{b_{j} t}-\lambda_{j}}\right)^{\mu_{j}} t^{m} \mid x^{n-1}\right\rangle \\
& +\left\langle\prod_{i=1}^{r}\left(\frac{t}{e^{a_{i} t}-1}\right)\left(\partial_{t} \prod_{j=1}^{s}\left(\frac{1-\lambda_{j}}{e^{b_{j} t}-\lambda_{j}}\right)^{\mu_{j}}\right) t^{m} \mid x^{n-1}\right\rangle \\
& +\left\langle\prod_{i=1}^{r}\left(\frac{t}{e^{a_{i} t}-1}\right) \prod_{j=1}^{s}\left(\frac{1-\lambda_{j}}{e^{b_{j} t}-\lambda_{j}}\right)^{\mu_{j}}\left(\partial_{t} t^{m}\right) \mid x^{n-1}\right\rangle .
\end{aligned}
$$

The third term of (26) is equal to

$$
\begin{aligned}
& m\left\langle\prod_{i=1}^{r}\left(\frac{t}{e^{a_{i} t}-1}\right) \prod_{j=1}^{s}\left(\frac{1-\lambda_{j}}{e^{b_{j} t}-\lambda_{j}}\right)^{\mu_{j}} t^{m-1} \mid x^{n-1}\right\rangle \\
& =m(n-1)_{m-1} B H_{n-m}(a ; b ; \lambda ; \mu) .
\end{aligned}
$$

The second term of (26) is equal to

$$
\begin{aligned}
& -\sum_{l=1}^{s} \frac{\mu_{l} b_{l}}{1-\lambda_{l}}\left\langle\prod_{i=1}^{r}\left(\frac{t}{e^{a_{i} t}-1}\right) \frac{1-\lambda_{l}}{e^{b_{l} t}-\lambda_{l}} \prod_{j=1}^{s}\left(\frac{1-\lambda_{j}}{e^{b_{j} t}-\lambda_{j}}\right)^{\mu_{j}} e^{b_{l} t} \mid t^{m} x^{n-1}\right\rangle \\
& =-(n-1)_{m} \sum_{l=1}^{s} \frac{\mu_{l} b_{l}}{1-\lambda_{l}}\left\langle\prod_{i=1}^{r}\left(\frac{t}{e^{a_{i} t}-1}\right) \frac{1-\lambda_{l}}{e^{b_{l} t}-\lambda_{l}} \prod_{j=1}^{s}\left(\frac{1-\lambda_{j}}{e^{b_{j} t}-\lambda_{j}}\right)^{\mu_{j}} e^{b_{l} t} \mid x^{n-m-1}\right\rangle \\
& =-(n-1)_{m} \sum_{l=1}^{s} \frac{\mu_{l} b_{l}}{1-\lambda_{l}} B H_{n-m-1}\left(b_{l} \mid a ; b ; \lambda ; \mu+e_{l}\right) .
\end{aligned}
$$

Since

$$
(n-1)_{l-1}(n-l)_{m}=(n-1)_{l+m-1}=(n-1)_{m-1}(n-m)_{l},
$$

the first term of (26) is equal to

$$
\begin{aligned}
& \left\langle\prod_{i=1}^{r}\left(\frac{t}{e^{a_{i} t}-1}\right) \prod_{j=1}^{s}\left(\frac{1-\lambda_{j}}{e^{b_{j} t}-\lambda_{j}}\right)^{\mu_{j}} t^{m} \mid \sum_{l=1}^{\infty}\left(\sum_{\nu=1}^{r} a_{v}^{l}\right) \frac{(-1)^{l-1} B_{l}}{l !} t^{l-1} x^{n-1}\right\rangle \\
& \quad=\sum_{l=1}^{n-m}\left(\sum_{\nu=1}^{r} a_{v}^{l}\right) \frac{(-1)^{l-1} B_{l}}{l !}(n-1)_{l-1}\left\langle\prod_{i=1}^{r}\left(\frac{t}{e^{a_{i} t}-1}\right) \prod_{j=1}^{s}\left(\frac{1-\lambda_{j}}{e^{b_{j} t}-\lambda_{j}}\right)^{\mu_{j}} t^{m} \mid x^{n-l}\right\rangle \\
& =\sum_{l=1}^{n-m}\left(\sum_{\nu=1}^{r} a_{v}^{l}\right) \frac{(-1)^{l-1} B_{l}}{l !}(n-1)_{l-1}(n-l)_{m} B H_{n-m-l}(a ; b ; \lambda ; \mu) \\
& \quad=(n-1)_{m-1} \sum_{l=1}^{n-m}(-1)^{l-1}\left(\begin{array}{c}
n-m \\
l
\end{array}\right) B_{l} B H_{n-m-l}(a ; b ; \lambda ; \mu) \sum_{\nu=1}^{r} a_{v}^{l} .
\end{aligned}
$$


Therefore, we get, for $n-1 \geq m \geq 1$,

$$
\begin{array}{rl}
(n)_{m} & B H_{n-m}(a ; b ; \lambda ; \mu) \\
= & m(n-1)_{m-1} B H_{n-m}(a ; b ; \lambda ; \mu) \\
& -(n-1)_{m} \sum_{j=1}^{s} \frac{\mu_{j} b_{j}}{1-\lambda_{j}} B H_{n-m-1}\left(b_{j} \mid a ; b ; \lambda ; \mu+e_{j}\right) \\
& +(n-1)_{m-1} \sum_{l=1}^{n-m}(-1)^{l-1}\left(\begin{array}{c}
n-m \\
l
\end{array}\right) B_{l} B H_{n-m-l}(a ; b ; \lambda ; \mu) \sum_{i=1}^{r} a_{i}^{l} .
\end{array}
$$

Dividing both sides by $(n-1)_{m-1}$, we obtain, for $n-1 \geq m \geq 1$,

$$
\begin{aligned}
(n- & m) B H_{n-m}(a ; b ; \lambda ; \mu) \\
= & -(n-m) \sum_{j=1}^{s} \frac{\mu_{j} b_{j}}{1-\lambda_{j}} B H_{n-m-1}\left(b_{j} \mid a ; b ; \lambda ; \mu+e_{j}\right) \\
& +\sum_{l=1}^{n-m}(-1)^{l-1}\left(\begin{array}{c}
n-m \\
l
\end{array}\right) B_{l} B H_{n-m-l}(a ; b ; \lambda ; \mu) \sum_{i=1}^{r} a_{i}^{l} .
\end{aligned}
$$

Thus, we get (25).

\subsection{A relation with Stirling numbers}

The Stirling numbers of the second kind $S_{2}(n, m)$ are defined by

$$
\frac{\left(e^{t}-1\right)^{m}}{m !}=\sum_{n=m}^{\infty} S_{2}(n, m) \frac{t^{n}}{n !}
$$

Then

$$
\phi_{n}(x):=\sum_{m=0}^{n} S_{2}(n, m) x^{m} \sim(1, \ln (1+t)) .
$$

\section{Theorem 6}

$$
B H_{n}(x \mid a ; b ; \lambda ; \mu)=\sum_{m=0}^{n} \sum_{l=m}^{n}\left(\begin{array}{l}
n \\
l
\end{array}\right) S_{1}(l, m) B H_{n-l} \phi_{m}(x) .
$$

Proof For (16) and (27), assume that $B H_{n}(x \mid a ; b ; \lambda ; \mu)=\sum_{m=0}^{n} C_{n, m} \phi_{m}(x)$. By (13), we have

$$
\begin{aligned}
C_{n, m} & =\frac{1}{m !}\left\langle\frac{1}{\prod_{i=1}^{r}\left(\frac{e^{a_{i} t}-1}{t}\right) \prod_{j=1}^{s}\left(\frac{e^{b_{j} t}-\lambda_{j}}{1-\lambda_{j}}\right)^{\mu_{j}}}(\ln (1+t))^{m} \mid x^{n}\right\rangle \\
& =\left\langle\prod_{i=1}^{r}\left(\frac{t}{e^{a_{i} t}-1}\right) \prod_{j=1}^{s}\left(\frac{1-\lambda_{j}}{e^{b_{j} t}-\lambda_{j}}\right)^{\mu_{j}} \mid \frac{1}{m !}(\ln (1+t))^{m} x^{n}\right\rangle \\
& =\left\langle\prod_{i=1}^{r}\left(\frac{t}{e^{a_{i} t}-1}\right) \prod_{j=1}^{s}\left(\frac{1-\lambda_{j}}{e^{b_{j} t}-\lambda_{j}}\right)^{\mu_{j}} \mid \sum_{l=m}^{\infty} S_{1}(l, m) \frac{t^{l}}{l !} x^{n}\right\rangle
\end{aligned}
$$




$$
\begin{aligned}
& =\sum_{l=m}^{n}\left(\begin{array}{l}
n \\
l
\end{array}\right) S_{1}(l, m)\left\langle\prod_{i=1}^{r}\left(\frac{t}{e^{a_{i} t}-1}\right) \prod_{j=1}^{s}\left(\frac{1-\lambda_{j}}{e^{b_{j} t}-\lambda_{j}}\right)^{\mu_{j}} \mid x^{n-l}\right\rangle \\
& =\sum_{l=m}^{n}\left(\begin{array}{l}
n \\
l
\end{array}\right) S_{1}(l, m) B H_{n-l} .
\end{aligned}
$$

Thus, we get identity (28).

\subsection{A relation with falling factorials}

\section{Theorem 7}

$$
B H_{n}(x \mid a ; b ; \lambda ; \mu)=\sum_{m=0}^{n} \sum_{l=m}^{n}\left(\begin{array}{l}
n \\
l
\end{array}\right) S_{2}(l, m) B H_{n-l}(x)_{m} .
$$

Proof For (16) and (17), assume that $B H_{n}(x \mid a ; b ; \lambda ; \mu)=\sum_{m=0}^{n} C_{n, m}(x)_{m}$. By (13), we have

$$
\begin{aligned}
C_{n, m} & =\frac{1}{m !}\left\langle\frac{1}{\prod_{i=1}^{r}\left(\frac{e^{a_{i} t}-1}{t}\right) \prod_{j=1}^{s}\left(\frac{e^{b_{j} t}-\lambda_{j}}{1-\lambda_{j}}\right)^{\mu_{j}}}\left(e^{t}-1\right)^{m} \mid x^{n}\right\rangle \\
& =\left\langle\prod_{i=1}^{r}\left(\frac{t}{e^{a_{i} t}-1}\right) \prod_{j=1}^{s}\left(\frac{1-\lambda_{j}}{e^{b_{j} t}-\lambda_{j}}\right)^{\mu_{j}} \mid \frac{1}{m !}\left(e^{t}-1\right)^{m} x^{n}\right\rangle \\
& =\left\langle\prod_{i=1}^{r}\left(\frac{t}{e^{a_{i} t}-1}\right) \prod_{j=1}^{s}\left(\frac{1-\lambda_{j}}{e^{b_{j} t}-\lambda_{j}}\right)^{\mu_{j}} \mid \sum_{l=m}^{\infty} S_{2}(l, m) \frac{t^{l}}{l !} x^{n}\right\rangle \\
& =\sum_{l=m}^{n}\left(\begin{array}{l}
n \\
l
\end{array}\right) S_{2}(l, m)\left\langle\prod_{i=1}^{r}\left(\frac{t}{e^{a_{i} t}-1}\right) \prod_{j=1}^{s}\left(\frac{1-\lambda_{j}}{e^{b_{j} t}-\lambda_{j}}\right)^{\mu_{j}} \mid x^{n-l}\right\rangle \\
& =\sum_{l=m}^{n}\left(\begin{array}{l}
n \\
l
\end{array}\right) S_{2}(l, m) B H_{n-l} .
\end{aligned}
$$

Thus, we get identity (29).

\subsection{A relation with higher-order Frobenius-Euler polynomials}

\section{Theorem 8}

$$
\begin{aligned}
& B H_{n}(x \mid a ; b ; \lambda ; \mu) \\
& \quad=\frac{1}{(1-\alpha)^{p}} \sum_{m=0}^{n}\left(\begin{array}{c}
n \\
m
\end{array}\right)\left(\sum_{l=0}^{p} \sum_{v=0}^{n-m}\left(\begin{array}{c}
p \\
l
\end{array}\right)\left(\begin{array}{c}
n-m \\
v
\end{array}\right)(-\alpha)^{p-l} l^{v} B H_{n-m-v}\right) H_{m}^{(p)}(x \mid \alpha) .
\end{aligned}
$$

Proof For (16) and

$$
H_{n}^{(p)}(x \mid \alpha) \sim\left(\left(\frac{e^{t}-\alpha}{1-\alpha}\right)^{p}, t\right),
$$


assume that $B H_{n}(x \mid a ; b ; \lambda ; \mu)=\sum_{m=0}^{n} C_{n, m} H_{m}^{(s)}(x \mid \alpha)$. By (13), similarly to the proof of (25), we have

$$
\begin{aligned}
& C_{n, m}=\frac{1}{m !}\left\langle\frac{\left(\frac{e^{t}-\alpha}{1-\alpha}\right)^{p}}{\prod_{i=1}^{r}\left(\frac{e^{a_{i} t}-1}{t}\right) \prod_{j=1}^{s}\left(\frac{e^{b_{j} t}-\lambda_{j}}{1-\lambda_{j}}\right)^{\mu_{j}}} t^{m} \mid x^{n}\right\rangle \\
& =\frac{\left(\begin{array}{c}
n \\
m
\end{array}\right)}{(1-\alpha)^{p}}\left\langle\prod_{i=1}^{r}\left(\frac{t}{e^{a_{i} t}-1}\right) \prod_{j=1}^{s}\left(\frac{1-\lambda_{j}}{e^{b_{j} t}-\lambda_{j}}\right)^{\mu_{j}} \mid\left(e^{t}-\alpha\right)^{p} x^{n-m}\right\rangle \\
& =\frac{\left(\begin{array}{c}
n \\
m
\end{array}\right)}{(1-\alpha)^{p}}\left\langle\prod_{i=1}^{r}\left(\frac{t}{e^{a_{i} t}-1}\right) \prod_{j=1}^{s}\left(\frac{1-\lambda_{j}}{e^{b_{j} t}-\lambda_{j}}\right)^{\mu_{j}} \mid \sum_{l=0}^{p}\left(\begin{array}{c}
p \\
l
\end{array}\right)(-\alpha)^{p-l} e^{l t} x^{n-m}\right\rangle \\
& =\frac{\left(\begin{array}{c}
n \\
m
\end{array}\right)}{(1-\alpha)^{p}} \sum_{l=0}^{p}\left(\begin{array}{c}
p \\
l
\end{array}\right)(-\alpha)^{p-l}\left\langle e^{l t} \mid \prod_{i=1}^{r}\left(\frac{t}{e^{a_{i} t}-1}\right) \prod_{j=1}^{s}\left(\frac{1-\lambda_{j}}{e^{b_{j} t}-\lambda_{j}}\right)^{\mu_{j}} x^{n-m}\right\rangle \\
& =\frac{\left(\begin{array}{l}
n \\
m
\end{array}\right)}{(1-\alpha)^{p}} \sum_{l=0}^{p}\left(\begin{array}{l}
p \\
l
\end{array}\right)(-\alpha)^{p-l}\left\langle e^{l t} \mid \sum_{\nu=0}^{\infty} B H_{v} \frac{t^{\nu}}{\nu !} x^{n-m}\right\rangle \\
& =\frac{\left(\begin{array}{l}
n \\
m
\end{array}\right)}{(1-\alpha)^{p}} \sum_{l=0}^{p}\left(\begin{array}{c}
p \\
l
\end{array}\right)(-\alpha)^{p-l} \sum_{v=0}^{n-m}\left(\begin{array}{c}
n-m \\
v
\end{array}\right) B H_{v}\left\langle e^{l t} \mid x^{n-m-v}\right\rangle \\
& =\frac{\left(\begin{array}{c}
n \\
m
\end{array}\right)}{(1-\alpha)^{p}} \sum_{l=0}^{p}\left(\begin{array}{c}
p \\
l
\end{array}\right)(-\alpha)^{p-l} \sum_{\nu=0}^{n-m}\left(\begin{array}{c}
n-m \\
v
\end{array}\right) B H_{\nu} l^{n-m-v} \\
& =\frac{\left(\begin{array}{c}
n \\
m
\end{array}\right)}{(1-\alpha)^{p}} \sum_{l=0}^{p}\left(\begin{array}{c}
p \\
l
\end{array}\right)(-\alpha)^{p-l} \sum_{\nu=0}^{n-m}\left(\begin{array}{c}
n-m \\
v
\end{array}\right) B H_{n-m-\nu} l^{\nu} .
\end{aligned}
$$

Thus, we get identity (30).

\subsection{A relation with higher-order Bernoulli polynomials}

Bernoulli polynomials $\mathfrak{B}_{n}^{(p)}(x)$ of order $p$ are defined by

$$
\left(\frac{t}{e^{t}-1}\right)^{p} e^{x t}=\sum_{n=0}^{\infty} \frac{\mathfrak{B}_{n}^{(p)}(x)}{n !} t^{n}
$$

(see e.g. [10, Section 2.2]).

\section{Theorem 9}

$$
B H_{n}(x \mid a ; b ; \lambda ; \mu)=\sum_{m=0}^{n}\left(\begin{array}{c}
n \\
m
\end{array}\right)\left(\sum_{l=0}^{n-m} \frac{\left(\begin{array}{c}
n-m \\
l
\end{array}\right)}{\left(\begin{array}{c}
n-m-l+p \\
p
\end{array}\right)} S_{2}(n-m-l+p, p) B H_{l}\right) \mathfrak{B}_{m}^{(p)}(x) .
$$

Proof For (16) and

$$
\mathfrak{B}_{n}^{(p)}(x) \sim\left(\left(\frac{e^{t}-1}{t}\right)^{p}, t\right)
$$


assume that $B H_{n}(x \mid a ; b ; \lambda ; \mu)=\sum_{m=0}^{n} C_{n, m} \mathfrak{B}_{m}^{(p)}(x)$. By (13), similarly to the proof of (25), we have

$$
\begin{aligned}
& C_{n, m}=\frac{1}{m !}\left\langle\frac{\left(\frac{e^{t}-1}{t}\right)^{p}}{\prod_{i=1}^{r}\left(\frac{e^{a_{i} t}-1}{t}\right) \prod_{j=1}^{s}\left(\frac{e^{b_{j} t}-\lambda_{j}}{1-\lambda_{j}}\right)^{\mu_{j}}} t^{m} \mid x^{n}\right\rangle \\
& =\left(\begin{array}{l}
n \\
m
\end{array}\right)\left\langle\prod_{i=1}^{r}\left(\frac{t}{e^{a_{i} t}-1}\right) \prod_{j=1}^{s}\left(\frac{1-\lambda_{j}}{e^{b_{j} t}-\lambda_{j}}\right)^{\mu_{j}} \mid\left(\frac{e^{t}-1}{t}\right)^{p} x^{n-m}\right\rangle \\
& =\left(\begin{array}{l}
n \\
m
\end{array}\right)\left\langle\prod_{i=1}^{r}\left(\frac{t}{e^{a_{i} t}-1}\right) \prod_{j=1}^{s}\left(\frac{1-\lambda_{j}}{e^{b_{j} t}-\lambda_{j}}\right)^{\mu_{j}} \mid p ! \sum_{l=0}^{\infty} S_{2}(l+p, p) \frac{t^{l}}{(l+p) !} x^{n-m}\right\rangle \\
& =\left(\begin{array}{c}
n \\
m
\end{array}\right) p ! \sum_{l=0}^{n-m} \frac{(n-m)_{l}}{(l+p) !} S_{2}(l+p, p)\left\langle\prod_{i=1}^{r}\left(\frac{t}{e^{a_{i} t}-1}\right) \prod_{j=1}^{s}\left(\frac{1-\lambda_{j}}{e^{b_{j} t}-\lambda_{j}}\right)^{\mu_{j}} \mid x^{n-m-l}\right\rangle \\
& =\left(\begin{array}{c}
n \\
m
\end{array}\right) \sum_{l=0}^{n-m} \frac{\left(\begin{array}{c}
n-m \\
l
\end{array}\right)}{\left(\begin{array}{c}
l+p \\
p
\end{array}\right)} S_{2}(l+p, p) B H_{n-m-l} \\
& =\left(\begin{array}{c}
n \\
m
\end{array}\right) \sum_{l=0}^{n-m} \frac{\left(\begin{array}{c}
n-m \\
l
\end{array}\right)}{\left(\begin{array}{c}
n-m-l+p \\
p
\end{array}\right)} S_{2}(n-m-l+p, p) B H_{l} .
\end{aligned}
$$

Thus, we get identity (32).

\section{Competing interests}

The authors declare that they have no competing interests.

\section{Authors' contributions}

All authors contributed equally to this work. All authors read and approved the final manuscript.

\section{Author details}

${ }^{1}$ Department of Mathematics, Sogang University, Seoul, 121-742, Republic of Korea. ${ }^{2}$ Department of Mathematics, Kwangwoon University, Seoul, 139-701, Republic of Korea. ${ }^{3}$ Graduate School of Science and Technology, Hirosaki University, Hirosaki, 036-8561, Japan.

\section{Acknowledgements}

The authors would like to thank the referees for their valuable comments. This work was supported by the National Research Foundation of Korea (NRF) grant funded by the Korea government (MOE) (No. 2012R1A1A2003786) and was partially supported by Kwangwoon University in 2014

\section{Received: 9 June 2014 Accepted: 11 August 2014 Published: 09 Sep 2014}

\section{References}

1. Araci, S, Acikgoz, M: A note on the Frobenius-Euler numbers and polynomials associated with Bernstein polynomials. Adv. Stud. Contemp. Math. 22, 399-406 (2012)

2. Ota, K: On Kummer-type congruences for derivatives of Barnes' multiple Bernoulli polynomials. J. Number Theory 92 , 1-36 (2002)

3. Kim, DS, Kim, T: Higher-order Frobenius-Euler and poly-Bernoulli mixed-type polynomials. Adv. Differ. Equ. 2013,251 (2013)

4. Kim, DS, Kim, T, Lee, S-H, Rim, S-H: A note on the higher-order Frobenius-Euler polynomials and Sheffer sequences. Adv. Differ. Equ. 2013, 41 (2013)

5. Can, M, Cenkci, M, Kurt, V, Simsek, Y: Twisted Dedekind type sums associated with Barnes' type multiple Frobenius-Euler /-functions. Adv. Stud. Contemp. Math. 18, 135-160 (2009)

6. Ryoo, CS, Song, H, Agarwal, RP: On the roots of the $q$-analogue of Euler-Barnes' polynomials. Adv. Stud. Contemp. Math. 9, 153-163 (2004)

7. Simsek, Y, Yurekli, $O$, Kurt, $\vee$ : On interpolation functions of the twisted generalized Frobenius-Euler numbers. Adv. Stud Contemp Math. 15, 187-194 (2007)

8. Kim, DS, Kim, T: Some identities of Frobenius-Euler polynomials arising from umbral calculus. Adv. Differ. Equ. 2012, $196(2012)$

9. Bayad, A, Kim, T: Results on values of Barnes polynomials. Rocky Mt. J. Math. 43, 1857-1869 (2013) 
10. Roman, S: The Umbral Calculus. Dover, New York (2005)

11. Kim, DS, Kim, T: Poly-Cauchy and Peters mixed-type polynomials. Adv. Differ. Equ. 2014, 4 (2014)

10.1186/1687-1847-2014-238

Cite this article as: Kim et al.: Barnes' multiple Bernoulli and generalized Barnes' multiple Frobenius-Euler mixed-type polynomials. Advances in Difference Equations 2014, 2014:238

Submit your manuscript to a SpringerOpen ${ }^{\odot}$ journal and benefit from:

- Convenient online submission

- Rigorous peer review

- Immediate publication on acceptance

- Open access: articles freely available online

- High visibility within the field

- Retaining the copyright to your article

Submit your next manuscript at $>$ springeropen.com 